\title{
ARTIGOS
}

\section{EL DUALISMO CARTESIANO Y SU RELACIÓN CON LA NUEVA MEDICINA A LA LUZ DE SU CORRESPONDENCIA*}

\author{
Alejandro Rojas Jiménez** \\ http://orcid.org/0000-0002-3503-3916 \\ rojas_a@uma.es \\ Pedro J. Chamizo Domínguez*** \\ http://orcid.org/0000-0003-3466-7711 \\ pjchd@uma.es
}

RESUMEN A pesar de que la reducción de R. Descartes de los animales a meras máquinas puede explicarse como una consecuencia lógica de su punto de partida metafísico y gnoseológico, se puede argüir que esta reducción parece muy difícil de defender, puesto que se nos antoja contradictoria con nuestra experiencia cotidiana. Por otra parte, uno de los propósitos de la filosofía de Descartes es la "conservación de la salud", que puede conseguirse mediante el establecimiento de una medicina "fundada en demostraciones infalibles". De cara a conseguir tal medicina el primer paso es alcanzar el conocimiento más preciso posible del cuerpo humano a fin de tener éxito en la práctica de la cirugía. De ahi que sea bastante plausible afirmar que la metafisica y la teoría del conocimiento de Descartes no serían sus principales intereses teóricos, sino solamente una explicación y justificación ingeniosas de lo que la ciencia de su tiempo llevaba haciendo no menos de cien años antes de él, así como una inteligente manera de obviar los escrúpulos cuando se anatomizaban cadáveres

* Artigo submetido em 27/03/2018 e aprovado em 07/05/2018.

** Universidad de Málaga. Málaga, Andalucía, España.

*** Universidad de Málaga. Málaga, Andalucía, España. 
humanos y animales (vivos). En consecuencia, en este artículo mantendremos que el mecanicismo y dualismo cartesianos son una verdadera filosofía de la ciencia, desde el momento en que ambos estaban dedicados a explicar y justificar lo que la ciencia estaba haciendo en la práctica.

Palabras clave Animales máquina, anatomía, cirugía, medicina, filosofía de la ciencia.

ABSTRACT Despite of the fact that $R$. Descartes's reduction of animals to mere machines can be explained as a logical consequence of his metaphysical and gnoseological starting point, one can argue that this reduction seems to be very difficult to defend, since it appears to be contradictory to our everyday experience. On the other hand, one of the explicitly confessed purposes of Descartes's philosophy is the "conservation of health", which can be achieved by establishing a medicine "founded in infallible demonstrations". The first step in order to get such medicine is to acquire the most accurate as possible knowledge of the human body in order to be successful in practicing surgery. That is why it is fairly plausible to state that Descartes's metaphysics and theory of knowledge were not his main theoretical concerns, but only a witty explanation and justification of what the science of his time was doing for no less than one century before him, as well as a clever manner to avoid scruples when anatomising both human corpses and (living) animals. Consequently, in this paper we will claim that Cartesian mechanicism and dualism are actual philosophies of science, given the fact that both were devoted to explain and justify what science was in practice doing.

Keywords Animal machines, anatomy, surgery, medicine, philosophy of science.

\section{Introducción}

Aunque Descartes no fuese exactamente el filósofo enmascarado que M. Leroy (1929) nos pintó, y que tendría una filosofía oculta distinta de la que publicó, no es menos cierto que no siempre explicitó en sus escritos publicados las razones últimas de lo que había dicho en ellos y, sobre todo, de lo que había dejado de decir. Hasta el punto de que el famoso larvatus prodeo, que Leroy convertirá en el lema e hilo conductor de su obra, aparece en unos escritos cartesianos tempranos - de 1619-a los que se les puso por título Cogitationes privatce (AT, X, p. 213). Si bien es cierto que sería excesivo hablar de una "doble 
filosofía" en Descartes, no es menos cierto que su abundante correspondencia nos muestra aspectos que quizás no hubiésemos podido inferir nunca de la obra publicada. Para ilustrar este extremo nos referiremos a dos cuestiones concretas. En primer lugar, la noticia de la condena de Galileo, que le hizo renunciar a dar la luz pública sus escritos en los que mantenía el movimiento de la Tierra. ${ }^{1} \mathrm{Y}$, en segundo lugar, su concepción provisional -y a pesar de que para Descartes la moral «est le dernier degré de la Sagesse» en la medida en que es una de las principales ramas del árbol de la ciencia junto con la medicina y la mecánica (Les principes de la philosophie AT, IX, p. 14) - ${ }^{2}$ de su doctrina moral, que nunca fue más allá de la «morale par provision» (Discours de la méthode AT, VI, p. 22), la cual no era más que una semblanza de la moral estoica clásica y de la que había propuesto M. de Montaigne, a pesar de que este último fuese la bestia negra de Descartes en otros muchos aspectos. ${ }^{3}$ Descartes no quiso ir más allá de esa moral provisional para que, entre otras cosas, «il n'y a point de matière d'où les malins puissent plus aisément tirer des prétextes pour calomnier» $(\grave{A}$ Chanut, 20 novembre 1647 AT, V, p. 87). ${ }^{4}$

Y uno de los varios aspectos sobre los que Descartes quizás no explicitase las razones últimas es el de la separación radical del ámbito de lo mental con respecto al ámbito de lo material en el ser humano. Nuestra propuesta en este trabajo va a consistir en postular que el dualismo cartesiano es la respuesta personal -y nunca explicitada- a los cambios que se habían producido en los siglos XVI y XVII en el campo de la medicina. Si esto fuese así, acontecería que la filosofía cartesiana sería un intento de respuesta a los problemas que habría planteado el avance de la medicina. Y este dualismo tuvo, como consecuencia colateral, el que hubiese de reducir los animales a meras máquinas.

1 «Mais je ne suis point si amoureux de mes pensées, que de me vouloir servir de telles exceptions, pour avoir moyen de les maintenir; et le désir que j'ay de vivre en repos et de continuer la vie que j'ay commencée en prenant pour ma devise : benè vixit, benè qui latuit, fait que je suis plus aise d'être délivré de la crainte que j'avais d'acquérir plus de connaissances que je ne désire, par le moyen de mon Écrit, que je ne suis fâché d'avoir perdu le temps et la peine que j'ay employée à le composer.» (À Mersenne, avril 1634 AT I, pp. 185186). En todas las citas de textos Descartes -así como en las del resto de autores franceses de los siglos XVI y XVII- hemos modernizado la grafía de acuerdo con las normas ortográficas del francés actual.

2 Prescindimos de analizar lo que de novedoso pudiera tener el símil cartesiano del árbol de la ciencia. Para una discusión detallada de este aspecto, ver (Ariew, 1992).

3 Y, precisamente, lo que más habría molestado a Descartes habría sido precisamente el que solamente se le hubiese dado la razón parcialmente, como señala É. Gilson muy gráficamente: "You can praise Montaigne by saying that he was partly right, not me. I was in the world to rid the world of Montaigne, don't you grant me the benefit of his indulgent scepticism, there is nothing that I hate more. I promised an infallible method; if I failed to fulfil completely my promise, I failed completely, say it» (Gilson, 1938, p. 152).

4 «Peut-être que, si j'y avais traité de la Morale, j'aurais occasion d'espérer qu'ils lui pourraient être plus agréables; mais c'est: de quoi je ne dois pas me mêler d'écrire. Messieurs les Régents font si animés contre moi, à cause des innocents principes de Physique qu'ils ont vus, et si en colère de ce qu'ils n'y trouvent aucun prétexte pour me calomnier, que, si je traitais après cela de la Morale, ils ne me laisseraient aucun repos» (À Chanut, 1er novembre $1646 \mathrm{AT}, \mathrm{IV}, \mathrm{p} .536)$. 


\section{La cirugía como parte esencial de la medicina}

Cuando se habla de la revolución científica que tuvo lugar en Europa en los siglos XVI y XVII se suele poner como modelo el ámbito de la astronomía y como la obra más representativa el De Revolutionibus Orbium Coelestium, de $\mathrm{N}$. Copérnico (1543). Hasta tal punto es esto cierto que el adjetivo copernicano se ha lexicalizado y ha pasado al lenguaje ordinario con el significado de «Dicho de un cambio en el comportamiento, en la manera de pensar, etc.: Radical o total» $(D E L, 2017) .{ }^{5}$ De modo análogo, el término revolución copernicana ha pasado a ser sinónimo de revolución científica/filosófica; al menos desde que I. Kant comparó lo que él mismo pretendía hacer en metafísica con lo que había hecho Copérnico en astronomía (Kant, 1919, p. 31). ${ }^{6}$ Ahora bien, el mismo año en que se publica el De Revolutionibus, se publica también otra obra que, aunque menos conocida fuera del ámbito de la medicina, ha significado tanto o más que la primera para el pensamiento occidental. Nos referimos al De humani corporis fabrica, de A. Vesalio.

La obra de Vesalio no es solamente relevante por sus aportaciones anatómicas originales (que no podían ser muchas sin el uso de instrumentos ópticos como el microscopio), sino también -y a la larga más provechosamente en nuestra opinión- por el papel que concede al conocimiento de la anatomía humana por parte de los médicos y por la misma concepción sobre cómo debe actuar un médico. A ambas cosas hace referencia en un texto que es, a la vez, crítico con las prácticas médicas de las que había sido testigo y programa de lo que debía ser un médico. En este programa la práctica quirúrgica se antoja como esencial:

Atque ita temporibus successu, curandi ratio tam misere divulsa est, ut medici quidem, se physicorum nomine venditantes, medicamentorum \& victus ad reconditos affectus præscriptionem sibi duntaxat arrogaverint, reliquam autem medicinam, iis quos Chirurgos nominant, vixque famulorum loco habent, relegarint, turpiter a se, quod præcipuum \& antiquissimum est medicinæ membrum (Vesalius, 1543, p. 3).

Esta forma de ejercer la medicina, criticada por Vesalio, consistía en separar la teoría de la práctica, de manera que, quienes tenían (los médicos) formación universitaria se limitaban a prescribir tratamientos sin tener contacto alguno con los pacientes, mientras que quienes tenían contacto con los pacientes

5 Y lo mismo que sucede en castellano, sucede también en inglés, donde el adjetivo Copernican también se ha lexicalizado en el lenguaje ordinario con el significado de "of radical or major importance or degree» (Merriam-Webster, 2017).

6 Por el contrario, a pesar de lo que se suele decir -e incluso escribir- Kant nunca utilizó la expresión kopernikanischen Wende/Revolution, aunque sí utilizó la expresión Umänderung der Denkart. 
(los barberos-cirujanos) carecían de cualquier formación teórica y anatómica (Carlino, 1999, pp. 39-42).

Este estado de cosas - del que aún quedan resquicios cuando distinguimos entre 'médicos' y 'practicantes' - es el que comenzará a cambiar cuando la cirugía se convierta en una disciplina inexcusable en la formación de todo médico. Este cambio, que se venía llevando a cabo en la Universidad de Padua desde hacía tiempo, se consolidará de forma definitiva cuando se cree la Universidad de Leiden en el año 1575. Esta universidad será en la que Petrus Paaw (Petrus Pavius) ocupó su primera cátedra de anatomía en 1589 y en la que, desde 1596/97, el propio Paaw hará exhibiciones anatómicas en el Anfiteatro Anatómico de Leiden, construido a imitación del de Padua creado en 1594 (Schumacher, 2007, p. 21). La mayor formación en anatomía por parte de los cirujanos redundará en el éxito de sus intervenciones, frente al escaso éxito curativo que tenían los médicos que no practicaban la cirugía. Esto hará que el prestigio de los cirujanos vaya cambiado en estos años hasta el punto de que Descartes liste a los cirujanos a la altura de los médicos, matemáticos y jurisconsultos «primæ notæ» (Ad G. Voetium AT, VIII, p. 150).

Para entender este cambio de paradigma en la consideración de la hasta entonces «poco noble» cirugía, ejercitada por curanderos, charlatanes y barberos, conviene prestar atención también a la figura de Ambroise Paré y al hecho de que en la guerra del Piamonte (1573) las armas de fuego fueran disparadas a corta distancia. Sería en la batalla de Dauvilliers cuando Paré utilizaría por primera vez la técnica de ligadura de arterias para tratar una amputación. Paré, que es considerado hoy como el padre de la cirugía, escribió antes de su Anatomie universelle du corps humain un texto que hace referencia justamente a este hecho capital que supuso la guerra, una vez más padre de todas las cosas («Пó $\lambda \varepsilon \mu \circ \varsigma$

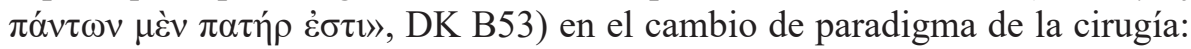
Méthode de traiter les plaies faites par les arquebuts et autres bastons à feu, et celles qui sont faites par la poudre à canon. El éxito de la cirugía está ligado de modo razonable a la capacidad de Paré de ligar arterias a los muñones en las amputaciones provocadas por armas de fuego. Si bien, habrá que esperar hasta el siglo XVIII para que se funde la Académie Royale de Chirurgie, concretamente en 1731, y para que se les prohíba a los barberos el ejercicio de la cirugía. Ya no será concebible el ejercicio de la medicina sin que el médico sea también cirujano, al menos entre los profesionales más punteros. Y, entre otras cosas, el ejercicio de la cirugía requiere de la formación más completa posible del médico-cirujano en anatomía.

Paré era un autor muy conocido y difundido en tiempos de Descartes (cfr., Wade, 2009), que nació sólo unos años después del fallecimiento de aquel. 
Si reparamos en ello podríamos llegar a pensar que aquella experiencia con miembros amputados que a la larga significaría el nacimiento de la cirugía moderna, podría estar detrás también -esta misma experiencia de Paré, quiero decir- de la filosofía del filósofo racionalista. Es sabido por todos que la experiencia con los miembros amputados ${ }^{7}$, en concreto con el hecho de que los pacientes podían sentir esos miembros amputados, llevó a una serie de intentos de explicación que podían haber sido continuados por Descartes en lugares claves de su obra filosófica, como por ejemplo, el siguiente pasaje de la sexta meditación:

\& quamvis toti corpori tota mens unita esse videatur, abscisso tamen pede, vel brachio, vel quâvis aliâ corporis parte, nihil ideo de mente subductum esse cognosco; neque etiam facultates volendi, sentiendi, intelligendi \&c. ejus partes dici possunt, quia una \& eadem mens est quae vult, quae sentit, quae intelligit. (Meditationes de Prima Philosophia AT, VIII, p. 86)

Esta propuesta de aproximación, por otro lado hoy incuestionable (cfr. Aucante, 2006, p. 47; cfr. Bitbol-Hespériès, 1990, p. 19), entre la filosofía de Descartes y los intelectuales (médicos y cirujanos) del momento, merece, y será el objeto de esta investigación, un análisis detenido y minucioso. Pues, de ser estas tesis comprobadas, posiblemente deban ser revisadas algunas de las clásicas interpretaciones cartesianas llevadas a cabo por la tradición filosófica, más preocupada por hacer una historia de las ideas que por entender el «paisaje» concreto (con más protagonismo de nervios, amputaciones y arterias que de textos filosóficos) de la obra cartesiana. No será nuestro propósito en este trabajo algo tan ambicioso como ese, sino tan sólo el anuncio anterior -ciertamente más modesto- de centrarnos en mostrar y fundamentar la importancia de esas preocupaciones «epocales» propias de cirujanos y médicos para entender el sentido filosófico de la obra cartesiana. Corresponderá al lector valorar las continuaciones y consecuencias posibles de este análisis. Si bien, una cosa parece clara: Descartes no fue médico, ni cirujano, de profesión.

7 «A most cleare and manifest argument of this false and deceiptful sense appears after the amputation of a member; for a long while after they will complaine of the part which is cut away. Verily it is a thing wondrous strange and prodigious, and which will scarce be credited, unlesse by such as have seen with their eyes, and heard with their ears the Patients who have many months after the cutting away of the Leg, grievously complained that they yet felt exceeding great pain of that leg so cut off» (A. Parey, 1649, p. 338). 


\section{III. «Je ne suis pas médecin de profession» (À Mersenne, 25 mai 1637 AT, I, p. 378)}

Aunque es cierto que Descartes no fue "médico de profesión" ni tampoco tuvo una formación académica en medicina, como reitera varias veces en su correspondencia ${ }^{8}$ no es menos cierto que bastantes años antes de publicar el Discurso del método, ya tenía en mente la posibilidad «de trouver une Médecine qui soit fondée en démonstrations infaillibles, qui est ce que je cherche maintenant» (À Mersenne, janvier 1630 AT, I, p. 106). Es más, incluso en los años 1632-1633 se le habría cursado una invitación para que ocupase una cátedra de medicina nada menos que en la Universidad de Bolonia (Manning, 2014). Esto permite aventurar la idea de que los aspectos metafísicos y gnoseológicos del pensamiento cartesiano no serían más que una actividad propedéutica para lo que verdaderamente le interesaría, que no sería otra cosa que el avance de la medicina. Y, aunque Descartes no hubiese sido un médico al uso, sí es probable que hubiese practicado el «exercice gratuit de la médecine, science occulte mise au service de l'humanité souffrante» (Adam, 1910, p. 48). Si esto es así, la presentación habitual que suele hacerse del pensamiento de Descartes, en la que priman los aspectos relacionados con "la filosofía primera", debería cambiarse por una presentación en la que se pensase a Descartes como un "filósofo de la ciencia" en la medida en que sus posturas metafísicas serían un intento de dar respuesta a problemas prácticos de la ciencia de su época.

Dicho de otra manera, Descartes no sería un metafísico que hace una determinada ciencia que es la consecuencia lógica de sus planteamientos metafísicos, sino que, por el contrario, su interés primordial habría sido dar una justificación a la ciencia que estaban haciendo sus contemporáneos. En consecuencia, nuestra tesis será justamente la siguiente: Descartes sería ante todo un "filósofo de la ciencia" cuya metafísica dualista no sería más que un intento de respuesta a los problemas que planteaba la práctica científica. Precisamente por ello, cuando Descartes cree que ha conseguido el método para un conocimiento seguro en las cuatro primeras partes del Discurso de método, es cuando podrá dedicar la quinta parte a «l'explication du mouvement du cœur et de quelques autres difficultés qui appartiennent à la Médecine» (Discours de la méthode AT, VI, p. 1). Y esto es lo que le permitirá confesar,

8 Por ejemplo, cuando declina hacer un diagnóstico sin ver al paciente «encore que je ne suis pas Docteur, je ne désespérerais pas pour cela d'y trouver remède; mais il faudrait être sur les lieux et voir le sujet» $(\dot{A}$ Mersenne, 11 mars 1640 AT, III, p. 42) o cuando, por el contrario, aventura un diagnóstico en una situación análoga a la anterior, «bien que je ne sois pas Médecin, l'honneur que votre Altesse me fit, l'été passé de vouloir savoir mon opinion, touchant une autre indisposition qu'elle avait pour lors, me fait espérer que ma liberté ne lui sera pas désagréable» (À Élisabeth, 18 mai 1645 AT, IV, p. 201). 
en el último parágrafo del Discurso, que su proyecto de vida a partir de ese momento no será otro que el de «n'employer le temps qui me reste à vivre à autre chose qu'à tâcher d'acquérir quelque connaissance de la Nature, qui soit telle qu'on en puisse tirer des règles pour la médecine, plus assurées que celles qu'on a eues jusques à présent» (Discours de la méthode AT, VI, p. 78. énfasis nuestro). Como resultado de este proyecto, ya en el mismo año 1637, Descartes confesará «je commence déjà tout de bon à étudier en médecine» ( $\grave{A}^{* * *}, 30$ août 1637 AT, I, p. 394), estudio que parece haber dado frutos con cierta rapidez en la medida en que solamente unos meses después asegure estar trabajando en "composer un abrégé de Médecine, que je tire en partie des livres, et en partie de mes raisonnements, duquel j'espère me pouvoir servir par provision à obtenir quelque délai de la nature, et ainsi poursuivre mieux ciaprès en mon dessein» (À Huygens, 25 janvier 1638 AT, I, p. 507). De manera que Descartes se convertirá así no solamente en un teórico de la ciencia, sino también en alguien que quiere que esa teoría tenga su correlato en la práctica cotidiana (Shapin, 2000).

Y este conocimiento de la naturaleza que nos permita sacar reglas para la medicina hay que conseguirlo, básicamente, mediante el estudio de la anatomía en cuanto conocimiento inexcusable para una cirugía eficiente. De ahí que, ya en una fecha tan temprana como es 1629 , justifique el retraso en escribir debido a su interés por iniciarse en el estudio de la anatomía: «je veux commencer à étudier l'anatomie [en Médecine et je n'écris presque rien]» ( $\grave{A}$ Mersenne, 18 décembre 1629 AT, I, p. 102). Y esta confesión cartesiana se nos antoja relevante no solamente por ser la indicación de su interés por la anatomía y la medicina y porque habría que tomarla al pie de la letra (Bitbol-Hespériès, 1990, p. 32; cfr. Aucante, 2006, pp. 12; 47), sino porque es signo de un giro que lleva a Descartes a interesarse especialmente por el estudio de los seres vivos como parte de la "física". Y la confesión de la intención de Descartes de estudiar anatomía parece que la llevará a cabo concienzudamente; pues, lo que no era más que la confesión de una mera intención en diciembre de 1629, parece estar llevándola a cabo en abril de 1630 . Y, además, asociando, al menos temporalmente si no también conceptualmente, el estudio de la anatomía al estudio de la química con objeto de encontrar remedio a la enfermedad:

J'étudie maintenant en chimie et en anatomie tout ensemble, et apprends tous les jours quelque chose que je ne trouve pas dedans les livres. Je voudrais bien être déjà parvenu jusques à la recherche des maladies et des remèdes, affin d'en trouver quelqu'un pour votre érésipèle, duquel je suis marri que vous êtes si longtemps affligé ( $\dot{A}$ Mersenne, 15 avril 1630 AT, I, p. 137). 
Y este estudio conjunto de la química y de la anatomía parece tener como objetivo no solamente el interés práctico consistente en la búsqueda de la cura de determinadas enfermedades, sino que parece tener un alcance teórico más amplio desde el momento en que, mediante el estudio de la anatomía de las cabezas de diversos animales, pretende nada menos que «expliquer en quoi consistent l'imagination, la mémoire, etc.» (À Mersenne, novembre/décembre 1632 AT, I, p. 263). La perseverancia de Descartes en el estudio de la anatomía es tal que todavía 10 años después de la primera confesión de su proyecto de estudiarla sigue diseccionando animales y enorgulleciéndose de haber superado al propio Vesalio en algunos aspectos, así como de que no hubiese muchos médicos que tuviesen sus conocimientos. ${ }^{9}$

\section{EI dualismo como respuesta}

La metafísica dualista cartesiana es por todos conocida. Lo que puede que no se haya difundido tan bien sea a qué responde este dualismo. La idea general es de hecho que el dualismo es una respuesta problemática, en tanto que plantea un problema de comunicabilidad entre la res cogitans y la res extensa. Pero cabría replantear la cuestión. Si hacemos caso al Descartes epistolar al que nos hemos referido en los apartados anteriores, más bien parece que se trata justamente de lo contrario: de que el dualismo es en sí mismo la respuesta a un problema. Queremos decir, que la solución fue separar tales sustancias porque resultaba un problema que no estuvieran separadas. Separarlas sería el modo de superar los «escrúpulos» que limitarían las prácticas en las que Descartes estaría interesado según lo expuesto en los parágrafos anteriores acerca de sus intereses en medicina y anatomía. Y así, dirá a Mersenne en 1639:

Et celui dont vous m'écrivez doit avoir l'esprit bien faible, de m'accuser d'aller par les villages, pour voir tuer des pourceaux; car il s'en tue bien plus dans les villes que dans les villages, où je n'ai jamais été pour ce sujet. Mais, comme vous m'écrivez, ce n'est pas un crime d'être curieux de l'Anatomie; et j'ai été un hiver à Amsterdam, que j'allais quasi tous les jours en la maison d'un boucher, pour lui voir tuer des bêtes, et faisais apporter de là en mon logis les parties que je voulais anatomiser plus à loisir; ce que j'ai encore fait plusieurs fois en tous les lieux où j'ai été, et je ne croie pas qu'aucun homme d'esprit m'en puisse blâmer (À Mersenne, 13 novembre 1639 AT, II, p. 621).

9 «J'ay considéré non seulement ce que Vezalius et les autres écrivent de l'Anatomie, mais aussi plusieurs choses plus particulières que celles qu'ils écrivent, lesquelles j'ai remarquées en faisant moi-même la dissection de divers animaux. C'est un exercice où je me suis souvent occupé depuis onze ans, et je croie qu'il n'y a guère de Médecin qui y ait regardé de si prés que moi.» (À Mersenne, 20 janvier 1639 AT, II, p. 525). 
En la imagen de sí mismo que nos da Descartes en este texto, y que recuerda más a la de un John Hunter (1728-1793) que a la de un filósofo al uso, ${ }^{10}$ se hace eco de esas acusaciones o reparos que sus intereses anatómicos le reportan. Pero si no son un crimen, como él dice, esas prácticas por las que es acusado por sus conciudadanos, es porque los animales sólo son res extensa, maquinaria sin res cogitans. Dichas prácticas, incluso con los humanos deberían ser permitidas y aceptadas, pues incluso en ellos sus cuerpos son sólo res extensa, y su res cogitans se encuentra a salvo de todas estas disecciones y estudios, como diría Aristóteles -salvando las expresiones propias de la distancia epocal-: separada.

En el caso de los animales el asunto se vuelve especialmente interesante, pues careciendo de res cogitans, carecen de cualquier experiencia pensante como la imaginación, la duda o incluso de vida sensitiva (cfr., Meditationes AT, VII, p. 28), y por ello mismo no es tampoco pensable que existan en ellos por ejemplo experiencia sensitiva del dolor (cfr. À Mersenne, 11 juin $1640 \mathrm{AT}$, III, p. 85), ya que éste no deja de ser justo eso: una expresión más de aquella vida sensitiva.

De hecho, Descartes, que sólo acepta como alma la parte racional (cfr., Descartes à Regius, mai 1641 AT, III, p. 371; cfr., Les Passions de l'Âme AT, $\mathrm{XI}$, p. 364) a la que también denomina como «mens» (Descartes à Regius, mai 1641 AT, III, p. 371), negará por ello que los animales tengan alma. Ellos tendrán, eso sí, una suerte de fuerza corpórea y sensitiva, vis motrix animalium. A la que se llegará a referir unos años más tarde como «anima corporea» ( $\dot{A}$ Morus, 5 fevrier 1649, AT, V, p. 276) ya que, a pesar de la oscuridad del término, en realidad dicha alma se identificaba ya en aquella carta a Regius del 41 con el mismo cuerpo.

Siendo así Descartes es contundente: entre un animal vivo y un animal muerto la diferencia es, teniendo como modelos las máquinas, simplemente de movimiento (cfr., Les Passions de l'Âme AT., XI, pp. 330-1), quiero decir, de poder o no poder moverse por sí mismo. Careciendo de alma corporal, todo su movimiento debe ser explicable por el cuerpo. Como no recordar aquí aquel

10 Nos referimos a la imagen tradicional del filósofo (al menos del racionalista) como alguien encerrado en sí mismo, alejado de la experiencia y que posiblemente tendría su origen en cierta imagen de Descartes, posiblemente la que nos brindaría Hegel en sus Vorlesungen über die Geschichte der Philosophie, en las que se analiza la metafísica cartesiana desligándola del paisaje real en el que se desarrolla y de sus intereses concretos. Así por ejemplo basa su concepción de Descartes en la siguiente idea: «Er ging davon aus, jede Voraussetzung müsse hintangesetzt werden, der Gedanke müsse von sich selbst anfangen; alles bisherige Philosophieren, besonders das von der Autorität der Kirche ausging, wurde hintangestellt» (Hegel, 1970, 20/125) y, de este modo lo convierte en un antecedente claro del idealismo alemán que él defiende: «Auch Fichte hat später mit derselben absoluten Gewißheit, mit dem Ich, wieder angefangen, ist aber dazu fortgegangen, aus dieser Spitze dann alle Bestimmungen zu entwickeln. Also diese Gewißheit ist das Prius» (Hegel, 1970, 20/131). 
«Etenim quid corpus possit, nemo hucusque determinavit» (Spinoza, Ethica, Pars III, Propositio 2, Scholium).

Descartes, además, tenía a su favor tanto a la tradición como a la propia ciencia del momento a la hora de proponer su dualismo. La filosofía Escolástica, que hablaba del spiritus animalis para explicar el movimiento en los animales, ejerce su influencia en la ciencia de la época y su interpretación de la sangre (cfr., H. Höffding, 1906, p. 243), por donde fluiría el llamado «espíritu animal». A su vez, la influencia del mecanicismo -dominador indiscutible del momento- que recibe de intelectuales reconocidos como Paré o el célebre De humani corporis fabrica, de Vesalio, permiten ver a Descartes como un continuador que, en lugar de proponer una doctrina sistemática alternativa acerca de los animales y de la interpretación mecanicista del cuerpo humano, busca más bien sumar criterios para apoyar unas prácticas médicas que todavía en ese momento despertaban recelo entre el vulgo, y que sólo la aceptación -coherente con la tradición y la ciencia del momento- de la insensibilidad de la res extensa podría salvar. Es decir, el dualismo cartesiano como respuesta a las prácticas científicas del momento, y no como problema generado ante la soledad de una estufa.

A menudo la clave del éxito de la filosofía tiene que ver con saber reconocer cuáles son los problemas y cuáles las respuestas, y sobre todo cuáles son los auténticos problemas a los que las distintas respuestas intentan responder. Es al menos la interpretación de la filosofía como ejercicio hermenéutico que, como mínimo después de Wahrheit und Methode, no queda más remedio que continuar.

Descartaríamos pues también la idea de que Descartes fuese un insensible. Si hacemos caso de las relativamente aún recientes afirmaciones de Finger, Descartes habría tenido un perro, al que habría llamado Monsieur Grat, y al que paseaba y trataba con cariño (cfr., Finger, 2000, p. 81). Cosa distinta es que, a juicio de Monsieur Descartes, y para el escándalo social de las personas del silgo XXI, fuese Grat el insensible, una mera e insensible res extensa.

\section{La insensibilidad de la res extensa}

Los estudios que Descartes estaba llevando a cabo antes de 1633 tenían como destino el libro que estaba pergeñando y que tendría como título Traité du monde et de la lumière. Pero la publicación de ese libro quedó en suspenso cuando Descartes tuvo noticias de la condena de Galileo de 1633 porque «quoique je pensasse qu'elles fussent appuyées sur des démonstrations très-certaines, et très-évidentes, je ne voudrais toutefois pour rien du monde les soutenir contre l'autorité de l'Église» (À Mersenne, avril 1634 AT, I, p. 285). La renuncia de Descartes a publicar su Traité du monde era, en principio, meramente provisional, 
pues en la misma carta confiesa al Mersenne no perder la esperanza en que, dado que la condena del heliocentrismo no había sido dictada por un concilio, pudiese ser revocada en el futuro como lo habían sido otras condenas análogas en el pasado: "et ainsi que mon Monde ne puisse voir le jour avec le temps; auquel cas j'aurai besoin moi-même de me servir de mes raisons» ( $\dot{A}$ Mersenne, avril 1634 AT, I, p. 288).

Como es sabido el Traité $d u$ monde quedaría inacabado y su publicación sería póstuma, pero, en compensación, Descartes va a dedicar sus esfuerzos a redactar la obra en la que va a establecer la exclusividad del cogito como lo esencialmente humano y que, por lo demás, no es otra cosa que «un ensayo cuyo estilo o genus dicendi imita la literatura de Montaigne, el famoso Discurso del método en que aparentemente no se habla una sola palabra de filosofía y poco más que ninguna de método» (Ortega y Gasset, 1944, XII/274). Y, aunque Ortega no aluda a esto, la razón por la que Descartes va a imitar la literatura de Montaigne, no solo estilísticamente sino incluso recurriendo a escribir en la misma lengua vehicular que había utilizado Michel de Montaigne, va a ser precisamente porque uno de los objetivos de Descartes -si no el principal objetivo- que se propone llevar a cabo consistirá en desmontar la tesis montaignista de que los animales tengan alguna clase de raciocinio.

Esta tesis Montaigne la había mantenido mediante el acto de habla indirecto de una pregunta en un famoso pasaje de su Apologie de Raymond Sebond:

Les arondelles, que nous voyons au retour du printemps fureter tous les coins de nos maisons, cherchent elles sans jugement et choisissent elles sans discrétion, de mille places, celle qui leur est la plus commode à se loger? (Montaigne, 1580, p. 432). ${ }^{11}$

$\mathrm{Y}$ este asunto es fundamental por cuanto que negar que los animales participen de la res cogitans permite, directamente, allanar el camino para hacer más llevadera la disección de animales, e incluso su vivisección, cosa que Descartes practicó en sus estudios de anatomía (Wollock, 2000, p. 418; Grayling, 2006, p. 159); e indirectamente, también permite obviar cualesquiera resquicios de escrúpulos que pudieran quedar a la altura del siglo XVII para la disección de cadáveres humanos con vistas a la formación de cirujanos. Y los

11 Hasta tal punto es razonable esta hipótesis, que la única vez que Descartes cita a Montaigne por su nombre es precisamente para mostrar su desacuerdo con respecto a este punto: «Pour ce qui est de l'entendement ou de la pensé que Montagne (sic) et quelques autres attribuent aux bêtes, je ne puis être de leur avis [...] Car, bien que Montagne (sic) et Charon (sic) aient dit qu'il y a plus de différence d'homme à homme, que d'homme à bête, il ne s'est toutefois jamais trouvé aucune bête si parfaite, qu'elle ait usé de quelque figne, pour faire entendre à d'autres animaux quelque chose qui n'eût point de rapport à ses passions» (Au Marquis de Newcastle, 23 novembre 1646 AT, IV, pp. 573-575). 
escrúpulos a los que nos referimos no vendrían -o no vendrían primordialmentede parte de las instituciones religiosas en razón de alguna creencia en el carácter sagrado del cuerpo humano, sino del hecho de que al anatomista y al médico se les permita hablar y escribir «of unclean things; because it is not to please, but profit» (Hobbes, 1904, p. 44). Y los reparos a esas "cosas sucias", sea esa suciedad de tipo físico o moral, ${ }^{12}$ parecen poder obviarse más fácilmente en la medida en que estemos tratando con una actividad cuyo objetivo sea la utilidad o el provecho. Y precisamente una de las cosas que Descartes cree que se puede conseguir con la aplicación práctica de su filosofía será su utilidad ${ }^{13}$ para

la conservation de la santé, laquelle est sans doute le premier bien et le fondement de tous les autres biens de cette vie; car même l'esprit dépend si fort du tempérament, et de la disposition des organes du corps que, s'il est possible de trouver quelque moyen qui rende communément les hommes plus sages et plus habiles qu'ils n'ont été jusques ici, je crois que c'est dans la médecine qu'on doit le chercher (Discours de la méthode AT, VI, p. 62. Énfasis nuestro).

De ahí que el propio Descartes confiese que el objetivo principal de sus estudios no sea otro que el de la conservación de la salud y el de descubrir cosas desconocidas hasta su momento en relación con este objetivo. Y para conseguir este objetivo tiene que superar los escrúpulos que pudiesen quedar con respecto a las prácticas anatómicas. Y el hecho de que en algún momento tenga que justificar las críticas que se le han dirigido por sus prácticas anatómicas es, plausiblemente, una prueba -no por indirecta menos fehaciente- de que sus contemporáneos aún tenían escrúpulos cuando se trataba de hacer anatomía. ( $A$ Mersenne, 13 novembre 1639 AT, II, p. 621). Y esto no solamente con respecto a la anatomía humana, sino también con respecto al estudio anatómico de los animales, que es muchas veces un medio muy precioso para el conocimiento de la propia anatomía humana. Esto hace que Descartes relacione el avance

12 Nos inclinamos a pensar que Hobbes es conscientemente ambiguo en este texto y que la "suciedad" a la que se refiere no es principalmente la suciedad física, sino la moral. Los traductores de este texto al castellano y al francés han mantenido la ambigüedad del original y han traducido ese texto como «sobre cosas sucias, porque su objeto no es agradar sino ser útil» (Hobbes, 2005, p. 57) y «sur des choses malpropres parce que son but n'est de plaire mais de servir» (Hobbes, 1921, p. 104), en castellano y francés, respectivamente. Pero el texto latino reza así: «de rebus obscænis [...], ut cui Finis est non placere, sed prodesse» (Hobbes, 1668, p. 36). Por su arte, la versión italiana ha desambiguado el texto original y concuerda con lo que dice el texto latino: «intorno ad argomenti indecenti, perchè non mirano al piacere, ma all'utile» (Hobbes, 1955, p. 105).

13 Precisamente por ello, cuando Pascal tenga que hacer una "enmienda a la totalidad" de la filosofía cartesiana después de haberle criticado a Descartes el haber recurrido a Dios porque no le quedaba más remedio, sentencie: «Descartes inutile et incertain» (Pascal, 1669, p. 1137, §195). 
de la medicina precisamente con el estudio de los animales, siendo el segundo una vía de acceso al primero. ${ }^{14}$

\section{Conclusión: EI Discurso cartesiano como herramienta para eludir escrúpulos que pudiesen surgir en la práctica de la anatomía}

Cuando alguien se enfrenta por primera vez a las tesis de Descartes sobre los animales y el que se les niegue no solamente el "alma racional" sino incluso cualesquiera sensaciones, la impresión de entrada es que Descartes estaría diciendo una de esas absurdidades sobre las que Cicerón ironizaba cuando comentaba la prohibición pitagórica de comer habas (De divinatione, 2.119) $)^{15}$ con una frase que el propio Descartes glosó e hizo suya. ${ }^{16}$ La primera impresión que se tiene, por tanto, cuando uno se encuentra con la tesis cartesiana de los animales-máquina, es que Descartes se aparta de lo razonable para proponer una idea del todo descabellada; idea que expondrá muy explícitamente $\mathrm{N}$. Malebranche cuando afirme que

dans les animaux il n'y a ni intelligence, ni âme, comme on l'entend ordinairement. Ils mangent sans plaisir, ils crient sans douleur, ils croissent sans le savoir, ils ne désirent rien, ils ne craignent rien, ils ne connaissent rien (Malebranche, 1674-1675, p. 394).

La tesis cartesiana sobre la "insensibilidad" de los animales ha hecho que sea agriamente criticado no solamente por los animalistas contemporáneos, sino también a lo largo de los siglos (cfr., Harrison, 1992, p. 219), comenzando por sus propios contemporáneos, como fue el caso de su corresponsal Henry More (latinizado, Morus) quien le llegó a confesar que la idea misma horrorizaba por letal y aniquiladora. ${ }^{17}$ La respuesta de Descartes a esta observación de More

14 «La conservation de la santé a été de tout temps le principal but de mes études, et je ne doute point qu'il n'y ait moyen d'acquérir beaucoup de connaissances, touchant la Médecine, qui ont été ignorées jusqu'à présent. Mais le Traité des animaux que je médite, et que je n'ai encore su achever, n'étant qu'une entrée pour parvenir à ces connaissances, je n'ai garde de me vanter de les avoir» (Au Marquis de Newcastle, octobre 1645 AT, IV, p. 329. Énfasis nuestro).

15 La frase literal de Cicerón es: «Sed nescio quo modo nihil tam absurde dici potest, quod non dicatur ab aliquo philosophorum» http://data.perseus.org/citations/urn:cts:latinLit:phi0474.phi053.perseus-lat2:2.119

16 Nótese que, en el original francés del Discurso del método, Descartes glosa la frase de Cicerón: «qu'on ne saurait rien imaginer de si étrange et si peu croyable, qu'il n'ait été dit par quelqu'un des Philosophes» (Discours de la méthode AT, VI, p. 16). Por el contrario, en la versión latina la cita casi literalmente: «nihil tam absurde dici posse (sic) quod non dicatur ab aliquo Philosophorum» (Dissertatio de methodo AT, VI, p. 548). Por lo demás, aunque la implicatura de ambos textos pueda ser la misma, esto es, que Descartes desautoriza las opiniones de los filósofos, las implicaciones son diferentes; puesto que, de que algo sea extraño o poco creíble no se implica necesariamente que, además, sea absurdo, esto es, que sea contrario a la razón.

17 «æque abhorret, ac ab internecina illa et iugulatrice sententia, quam in Methodo tulisti, brutis omnibus vitam sensumque eripiens» (À Morus, 11 de décembre 1648 AT, V, p. 243). 
será la de distinguir entre 'vida' y 'sentido', siendo el caso que Descartes dirá que no niega la primera, aunque sí el segundo, amén del pensamiento. Incluso acepta el que los animales tengan "sensación", si tal cosa se explica de forma mecanicista. Pero lo más relevante del texto cartesiano en defensa de sus tesis frente a los reparos de More consiste en argumentar que su postura no debe entenderse tanto como crueldad para con los animales, sino, más bien, como compasiva para con los humanos, quienes tienen que comerlos y matarlos:

Velim tamen notari me loqui de cogitatione, non de vita, vel sensu: vitam enim nulli animali denego, utpote quam in solo cordis calore consistere statuo; nec denego etiam sensum, quatenus ab organo corporeo dependet. Sicque haec mea opinio non tam crudelis est erga belluas, quam pia erga homines, Pythagoreorum superstitioni non addictos, quos nempe a criminis suspicione absoluit, quoties animalia comedunt vel occidunt (À Morus, 5 février 1649 AT, V, pp. 278-279).

Ahora bien, llegados a este punto cabe preguntarse si este cambio tan radical de nuestra intuición natural -que nos hace estar más cercanos de Montaigne que de Descartes- fue la consecuencia de sus planteamientos metafísicos dualistas y mecanicistas o si, por el contrario, y como sospechó Ortega, ${ }^{18}$ no habría sido más bien al revés. Esto es lo que habríamos intentado defender y mostrar en este trabajo, que Descartes habría ideado su dualismo, su mecanicismo y su tesis que reduce los animales a ser meras máquinas como un constructo teórico para justificar lo que él y sus contemporáneos estaban haciendo y para lo que no servían ni la tradición filosófica aristotélico-escolástica, ni el escepticismo de la segunda mitad del siglo XVI. Con respecto a la tradición aristotélico-escolástica, Descartes tiene que desligarse de ella precisamente por su sensualismo; ${ }^{19}$ mientras que el escepticismo, como hemos apuntado anteriormente, era el enemigo que había que batir, no solamente porque también era sensualista, sino, además, por rechazar la posibilidad de que el hombre alcance certezas absolutas.

Aunque paradójicamente, la primera prueba que Descartes puede presentar de su mecanicismo y de su dualismo le va a venir de la mano de un aristotélico como era el caso de William Harvey y la explicación mecánica que proporciona

18 «Una inversión tan completa de la perspectiva espontánea no fue en Descartes y en las generaciones siguientes un resultado imprevisto a que súbitamente se llega en vista de ciertas pruebas. Al contrario, se comienza por desear, más o menos, confusamente, que las cosas sean de una cierta manera, y luego se buscan las pruebas para demostrar que las cosas son, en efecto, como nosotros deseábamos. Con esto no quiero, en manera alguna, decir que las pruebas sean ilusorias: es, simplemente, hacer constar que no son las pruebas quienes nos buscan y asaltan, sino nosotros los que vamos a buscarlas, movidos por previos afanes» (Ortega y Gasset, 1923, pp. 160-161).

19 «Descartes pelea a muerte con Aristóteles y el escolasticismo porque son sensualistas. La cruzada cartesiana va contra el conocimiento sensible, quiere liberar al hombre de su esclavitud sensorial» (Ortega y Gasset, 1937, p. 274). 
de la circulación de la sangre en su Exercitatio Anatomica de Motu Cordis et Sanguinis in Animalibus Exercitatio Anatomica de Motu Cordis et Sanguinis in Animalibus (1628). Más allá de las diferencias en las interpretaciones sobre cuál fuese la causa del movimiento de la sangre entre Harvey y Descartes (Gorham, 1994; González Recio, 1995; Laguna, 2015), lo cierto es que la explicación de la circulación de la sangre, de Harvey, «auquel il faut donner la louange d'avoir rompu la glace en cet endroit» (Discours de la méthode AT, VI, p. 51), fue publicada 9 años antes que el Discurso del método. Y no es del todo irrazonable pensar que el dualismo del Discurso del método no fuese más que una manera ingeniosa de eludir cualesquiera escrúpulos que pudiesen surgir en la práctica de la anatomía. A menos que aceptemos acríticamente el relato de la escena de la estufa, según el cual Descartes se habría revelado a sí mismo su esencia como ser pensante.

\section{Referencias bibliográficas}

a) Bibliografía citada de Descartes:

Las obras de Descartes citadas en el texto, y que se indican a continuación, han sido citadas según la edición de referencia: la Euvres editada por Adam y Paul Tannery en los años 1897-1913 y publicadas en París por Léopold Cerf.

À**, 30 août 1637, AT. I.

À Chanut du ler novembre 1646, AT. IV.

À Descartes, 11 de décembre 1648, AT. V.

À Élisabeth, 18 mai 1645. AT. IV.

À Huygens, 25 janvier 1638. AT. I.

À Mersenne, 11 juin 1640, AT. III.

À Mersenne, 11 mars 1640, AT. III.

À Mersenne, 13 novembre 1639, AT. II.

À Mersenne, 20 janvier 1639, AT. II.

À Mersenne, avril 1634, AT. I.

À Mersenne, novembre/décembre 1632, AT. I.

À Morus, 5 février 1649, AT. V.

À Regius, mai 1641, A. T. III.

Ad G. Voetium

Au Marquis de Newcastle, 23 novembre 1646. AT. IV.

Au Marquis de Newcastle, octobre 1645. AT. IV.

Discours de la méthode. AT. VI.

Les Passions de l'Ame, AT. XI.

Meditationes de Prima Philosophia, AT. VII. 
b) Otra bibliografía secundaria utilizada:

ADAM, CH. "Vie \& Euvres de Descartes. Étude historique”. Supplément à l'Edition de Descartes, T. XII. Paris: Léopold Cerf, 1910.

ARIEW, R. "Descartes and the Tree of Knowledge". Synthèse, 92/1, pp. 101-116, 1992. AUCANTE, V. "La philosophie médicale de Descartes". Paris: Presses Universitaires de France, 2006.

BITBOL-HESPERIES, A. "Le principe de vie chez Descartes". Paris: J. Vrin, 1990. CARLINO, A. "Books of the Body. Anatomical Ritual and Renaissance Learning". Traducción inglesa de John Tedeschi y Anne C. Tedeschi. Chicago: The University of Chicago Press, 1999.

DIELS, H., KRANZ, W. "Die Fragmente der Vorsokratiker". Weidmannsche Buchhandlung, Berlin, 1906.

FINGER, S. "Minds behind the Brain: A History of the Pioneers and Their Discoveries". Oxford: University Press, 2000.

GILSON, É. "The Unity of Philosophical Experience". London: Sheed and Ward, 1938. GONZÁLEZ RECIO, J. "La paradoja Harvey-Descartes y el proyecto de una biología teórica”. Thémata: Revista de filosofia, 14, pp. 62-82, 1995.

GORHAM, G. "Mind-Body Dualism and the Harvey-Descartes Controversy". Journal of the History of Ideas, 55/2, pp. 211-234, 1994.

GRAYLING, A. C. "Descartes: The Life of Rene Descartes and Its Place in His Times." New York: Simon \& Schuster, 2006.

HARRISON, P. "Descartes on Aniamls". The Philosophical Quarterly, 42/167, pp. 229-227, 1992.

HEGEL, G.W.F. (1840-1844). "Vorlesungen über die Geschichte der Philosophie", Berlin: Duncker und Humbolt. In: G.W.F. Hegel. Werke in 20 Bänden. Suhrkamp, Berlín, 1970.

HOBBES, Th. (1651). "Leviathan, or the Matter, Forme \& Power of Commonwealth, Ecclesiasticall and Civill”. Ed. de A. R. Waller. Cambridge: Cambridge University Press, 1904.

HOBBES, Th. (1651). "Leviathan ou la Matière, la Forme et la Puissance d'un État ecclésiastique et civil". Traduction française en partie double d'après les textes anglais et latin originaux par R. Anthony. Paris: Marcel Giard, 1921.

HOBBES, Th. (1651). "Il Liviatano". Traduzione, introduzione e note a cura di Roberto Giammanco. Torino: Unione Tipografico-editrice Torinense, 1955.

HOBBES, Th. (1651). "Leviatán. O la materia, forma y poder de una república eclesiástica y civil”. Traducción de Manuel Sánchez Sarto. Buenos Aires: Fondo de Cultura Económica, 2005.

HOBBES, Th. (1651). "Leviathan, sive De Materia, Forma, \& Potestate Civitatis Ecclesiasticæ et Civilis". Amstelodami: Apud Ioannem Blaev, 1668.

HÖFFDING, H. "Histoire de la philosophie", I. Paris: Félix Alcan, 1906.

KANT, I. (1787). "Kritik der reinen Vernunft". Neu Herausgegeben von Theodor Valentiner. Leipzig: Verlag von Felix Meiner, 1919. 
LAGUNA, R. "Mecanicismo o vitalismo. La polémica Descartes-Harvey sobre el movimiento del corazón". Protrepsis, 4/7-8, pp. 6-19, 2015.

LEROY, M. "Descartes, le philosophe au masque". Paris: Rieder, 1929.

MALEBRANCHE, N. (1674-1675). "De la recherche de la vérité. Où l'on traite de la Nature de l'Esprit de l'homme, \& de l'usage qu'il en doit faire pour éviter l'erreur dans les Sciences, en Euvres Complètes", I. Édition établie par Geneviève Rodis-Lewis avec la collaboration de Germain Malbreil. Paris: Gallimard, 1979.

MANNING, G. "Descartes and the Bologna affair". British Journal of History of Science, 47/1, pp. 1-13, 2014.

MERRIAM-WEBSTER. "Merriam-Webster Dictionary". Disponible en: http://www. merriam-webster.com/, 2017.

MONTAIGNE, M. de. (1580). "Essais, en CEuvres Complètes". Textes établis par Albert Thibaudet et Maurice Rat. Paris: Gallimard, 1976.

ORTEGA Y GASSET, J. (1923). "El tema de nuestro tiempo". In: Obras completas III. Madrid: Alianza-Revista de Occidente, 1983.

ORTEGA Y GASSET, J. (1937). "Bronca en la física I. Una polémica en la religión más pacífica". In: Obras completas $V$. Madrid: Alianza-Revista de Occidente, 1983.

ORTEGA Y GASSET, J. (1944). “La razón histórica, en Obras completas XII”. Madrid: Alianza-Revista de Occidente, 1983.

PAREY, A. "The Works of that Famous Chirurgion". London: Clarke, 1649.

PASCAL, B. (1669). "Pensées, en CEuvres complètes". Texte établi et annoté par Jacques Chevalier. Paris: Gallimard, 1976.

SCHUMACHER, G-H. "Theatrum Anatomicum in History and Today". International Journal of Morphology, 25/1, pp. 15-32, 2007.

SHAPIN, S. "Descartes the doctor: Rationalism and its therapies". British Journal of History of Science, 33/2, pp. 131-154, 2000.

SPINOZA, B. "Ethica ordine geometrico demonstrata". In: Spinoza Opera, II. Heidelberg Akademie der Wissenschaften: Carl Gebhardt, Winters Universitätsbuchhandlung, 1972. VESALIUS, A. "De humani corporis fabrica libri septem". Basileae: ex officina Ioannis Oporini, 1543.

WADE, N. J. "Beyond body experiences: Phantom limbs, pain and the locus of sensation", Cortex, 45/2, pp. 243-255, 2009.

WOLLOCK, N. "Christiaan Huygens's Attitude toward Animals". Journal of the History of Ideas, 61/3, pp. 415-432, 2000. 